\title{
Sistematizando o conhecimento acerca da prevenção das lesões do tipo skin tears na pele senil
}

\section{Systematizing knowledge about prevention of skin tears in the aged skin}

\author{
Beatriz Espindola Gomes ${ }^{1} \bullet$ Priscila Vieira de Souza ${ }^{2} \bullet$ Gustavo Dias da Silva $\bullet$ Raí Moreira Rocha $\bullet$ \\ Sergio Noboru Kuriyama ${ }^{5}$ Zenith Rosa Silvino ${ }^{6}$
}

\section{RESUMO}

Com o objetivo de identificar, na literatura científica, as melhores intervenções para prevenir o surgimento das lesões por fricção do tipo skin tears no idoso, este estudo adotou um método de revisão integrativa das produções científicas disponíveis nas bases de dados da Pubmed (Public MEDLINE - Literatura Internacional em Ciências da Saúde) e na Biblioteca Virtual em Saúde (BVS). Resultados: Após a busca, seleção e análise prévia dos artigos, 12 foram selecionados para compor a amostra, a maior parte $(73,5 \%)$ produzidos por enfermeiros, todos publicados em inglês, sendo $50 \%$ estadunidenses e nenhum brasileiro. Conclusões: Foram agrupadas quatro esferas de cuidado para prevenção das lesões do tipo skin tears: manter a homeostase cutânea no processo do envelhecimento; evitar acidentes com a pele no contexto da senilidade; proteger a pele senil do trauma mecânico; sistematizar e educar para o cuidado com a pele do idoso.

Palavras-chave: Lesões; Atenção à Saúde do Idoso; Enfermagem..

\begin{abstract}
In order to identify the scientific literature the best interventions to prevent the onset of lesions friction type skin tears in the elderly, this study adopted a method of integrative review of the available scientific production in the databases of PubMed (MEDLINE Public - International Literature Health Sciences) and VHL (Virtual Health Library). Results: After the search, selection and analysis of previous articles, 12 were selected for the sample, the majority (73.5\%) produced by nurses, all published in English, with $50 \%$ and no Brazilian Americans. Conclusions: Four spheres of care for injury prevention type of skin tears were grouped: maintaining skin homeostasis in the aging process; prevent injury to the skin in the context of senility; protect the senile skin mechanical trauma; organize and educate for the skin care of the elderly.
\end{abstract}

Keywords: Injuries; Health care of the elderly; Nursing.

${ }^{3}$ Enfermeiro. Doutorando em Ciências do Cuidado em Saúde pela Universidade Federal Fluminense. E-mail: gustavodias@me.ufrj.br

${ }^{4}$ Enfermeiro. Mestrando em Ciências do Cuidado em Saúde.Professor no Instituto Superior de Educação e Saúde Gênesis. Niterói, RJ, Brasil. E-mail: moreirarocha958@gmail.com ${ }^{5}$ Biólogo - UERJ. Mestre em Biociência Nuclear - UERJ. Doutor em Toxicologia - CUMS - Berlin. Pós-doc em Toxicologia Ambiental - FIOCRUZ. Rio de Janeiro (RJ), Brasil. E-mail: sergio.kuriyama@gmail.com

${ }^{6}$ Enfermeira. Doutora em Enfermagem. Professora Titular do Departamento de Fundamentos de Enfermagem e Administração Escola de Enfermagem Aurora de Afonso Costa EEAAC/UFF. Niterói (RJ), Brasil. E-mail: zenithrosa@terra.com.br

Os autores declaram não haver conflitos de interesse nem fontes de financiamento. 


\section{INTRODUÇÃO}

As lesões por fricção, conhecidas como skin tears, são feridas traumáticas que ocorrem principalmente nas extremidades dos idosos, resultantes da fricção ou de uma combinação de fricção e cisalhamento, levando à separação da epiderme da derme ou separando totalmente a epiderme e a derme das estruturas subjacentes. Esta lesão é consequência obrigatória de um traumatismo mecânico que produz um rasgo de pele ${ }^{1}$ e ocorre especialmente em idosos, devido às características peculiares que aparecem por conta do processo do envelhecimento.

Nos Estados Unidos, ocorrem mais de 1,5 milhão de lesões do tipo skin tears a cada ano em idosos internados em centros de saúde ${ }^{2}$; estima-se que, até 2030, o número de indivíduos de alto risco para essas lesões seja de 8,1 milhões de pessoas, somente nesse país ${ }^{3}$.

Estudos preliminares sugerem que lesões do tipo skin tears podem ser mais prevalentes do que as úlceras por pressão $0^{4}$. Comparadas às úlceras por pressão, mais extensas e dispendiosas, as lesões do tipo skin tears são muitas vezes consideradas menores e sem importância, quando, na realidade, essas feridas são dolorosas e podem levar a complicações como infecções e sangramentos ${ }^{5}$. Quase $80 \%$ das lesões do tipo skin tears ocorrem nos braços e mãos, mas podem ocorrer em qualquer parte do corpo, como nas nádegas e nas costas, onde podem ser confundidas com úlceras por pressão estágio $\|^{6}$.

\section{REVISÃO DA LITERATURA}

A pele é o maior órgão do nosso organismo, tendo em média, no adulto, uma área total de 2 metros quadrados; pesa aproximadamente $2,7 \mathrm{~kg}$ e recebe $1 / 3$ do volume de sangue circulante; é ativa e suas funções são vitais para manutenção da homeostase. Como revestimento externo do corpo, forma uma barreira protetora contra ferimentos e defesa contra organismos patógenos, ao mesmo tempo em que auxilia na regulação hídrica e excreção de produtos metabolizados, além de participar da regulação da temperatura corporal e conter terminações nervosas sensitivas. É composta por três camadas: a epiderme, a derme e a hipoderme ${ }^{7}$.

A epiderme é um epitélio avascular plano nutrido por meio da difusão de substâncias desde o leito capilar da derme, com queratinócitos cilíndricos que fazem mitose e regeneram a pele sob controle específico de substâncias, como os fatores de crescimento, hormônios e vitaminas. Está em constante processo de cornificação ou queratinização e é composta por cinco camadas celulares diferentes: basal ou germinal, espinhosa, granulosa, lúcida e córnea, sendo a camada basal a mais profunda da epiderme, ondulada e com invaginações coniformes, chamadas papilas da derme, recoberta pela membrana basal que limita a epiderme e a derme. Esta camada interna tem a função de fixar as células basais realizando força de ancoragem entre as estruturas de ligação entre a epiderme e a derme ${ }^{8}$.
A derme está unida à membrana basal, formada de tecido conjuntivo vascularizado e com abundantes terminações nervosas. Possui glândulas sebáceas cujos grânulos contêm substâncias, como heparina e histamina, macrófagos, linfócitos e fibroblastos que sintetizam e liberam os precursores do colágeno, especialmente o do tipo I, elastina, entre outras que compõem uma substância básica gelatinosa de matriz extracelular e dão à pele alta capacidade de resistência e elasticidade ${ }^{8}$.

A hipoderme é a camada mais profunda da pele, composta por tecido conjuntivo frouxo, que se une à fáscia muscular ou ao periósteo, e tecido adiposo com função de armazenamento, isolamento e modelação8.

Durante o processo senil, ocorre o aumento da queratinização prematura da epiderme, alargamento e aplanamento dos sulcos intrapapilares e a perda do preguiamento da lâmina basal, de modo que o sistema de ancoragem existente entre a epiderme e derme passa a não suportar de maneira eficaz a fricção e o cisalhamento. Observa-se, então, a redução da produção do colágeno tipo I e o aumento do tipo III pelos fibroblastos, caracterizando a situação da derme no processo de envelhecimento ${ }^{9-11}$.

Acontece também a desorganização das fibras de colágeno que se tornam menos densas, fragmentadas e separadas por grandes áreas ocupadas por substância fundamental. Os micropolissacarídeos perdem sua função estrutural e apresentam-se como um sedimento disforme; com isso, a pele perde força tênsil, resistência e elasticidade. Ocorre uma redução na produção das glândulas sudoríparas e sebáceas, levando a pele a uma condição de desidratação, ressecamento e perda da elasticidade. A hipoderme tornase mais delgada, e, consequentemente, a camada adiposa não suporta a absorção de impacto como antes ${ }^{9-11}$.

Depois de duas décadas de pesquisas, está agora claramente reconhecido que as lesões do tipo skin tears em idosos ocorrem devido, em parte, às mudanças fisiológicas da pele à medida que envelhecemos. Estas características adquiridas pela pele ao decorrer dos anos, associadas ao fato de que existe uma menor irrigação sanguínea da pele por conta da priorização do fluxo aos órgãos nobres, predispõem o surgimento das lesões do tipo skin tears nestes pacientes, ao menor trauma por fricção ou a combinação de fricção e cisalhamento ${ }^{11-12}$.

Apesar da magnitude do problema, gerada pela alta incidência da lesão entre idosos em todo o mundo, a temática ainda é pouco conhecida e explorada,em especial, no Brasil. 0 primeiro estudo brasileiro abordando o tema skin tear - uma dissertação de mestrado defendida por uma enfermeira na Escola de Enfermagem da Universidade de São Paulo - data de $2010^{11}$.

O manejo adequado de pacientes com lesões do tipo skin tears é um desafio permanente para os profissionais da saúde ${ }^{13}$. Por tratar-se de uma lesão dolorosa, de alta incidência, diferenciada, pouco divulgada e pouco conhecida no Brasil, de difícil avaliação e que pode provocar 
inúmeras complicações, faz-se necessário conhecer o estado da arte desta temática a fim de fornecer subsídios para fundamentar a prática de enfermagem no que tange a avaliação, prevenção e tratamento destas lesões.

Torna-se ainda mais importante o fomento e divulgação do tema, quando se avalia a proporção de indivíduos suscetíveis à lesão devido ao acentuado envelhecimento da população mundial e do Brasil. Segundo dados do IBGE, em 2008, para cada grupo de 100 crianças de 0 a 14 anos existiam 24,7 idosos de 65 anos ou mais. Em 2050, a projeção é de que para cada 100 crianças de 0 a 14 anos existirão 172,7 idosos $^{14}$.

Deste modo, conduziu-se o presente estudo com o objetivo de identificar, na literatura científica, as melhores intervenções para prevenir o surgimento das lesões do tipo skin tears no idoso.

\section{MÉTODO}

$\mathrm{Na}$ construção deste trabalho, para conhecimento das melhores práticas de assistência de enfermagem voltadas para a prevenção das skin tears no idoso, foi empregado o método de revisão integrativa da literatura (RIL).

A revisão integrativa inclui a análise de pesquisas relevantes que dão suporte para a tomada de decisão e a melhoria da prática clínica. Possibilita a síntese do estado do conhecimento de um determinado assunto, de maneira sistemática e ordenada, contribuindo para o aprofundamento do conhecimento do tema investigado, além de apontar lacunas do conhecimento que precisam ser preenchidas com a realização de novos estudos ${ }^{15-16}$.

Como um dos métodos de pesquisa utilizados na Prática Baseada em Evidências (PBE), a revisão integrativa permite a incorporação de evidências na prática de enfermagem prestada nos diversos níveis de atenção, reforçando a importância da pesquisa para a prática clínica ${ }^{16}$.

Conduzida de modo a identificar, analisar e sintetizar resultados de estudos independentes sobre o mesmo assunto, a revisão integrativa pode ser composta por seis etapas: elaboração da pergunta norteadora, busca ou amostragem na literatura, coleta de dados, análise crítica dos estudos incluídos, discussão dos resultados e apresentação da revisão integrativa ${ }^{17}$.

A questão norteadora que acompanhou este caminho investigativo foi: "Quais são as medidas preventivas evidenciadas na literatura como melhores práticas para prevenção das lesões do tipo skin tears no idoso?".

Para realizar a busca dos estudos, foi acessada a base de dados da PubMed e a Biblioteca Virtual em Saúde (BVS), incluindo a Literatura Latino-Americana e do Caribe em Ciências da Saúde (LILACS), Índice Bibliográfico Espanhol em Ciências da Saúde (IBECS), Literatura Internacional em Ciências da Saúde (MEDLINE), Revisões Sistemáticas da Colaboração Cochrane (COCHRANE) e Scientific Electronic Library Online (SciELO). Foram utilizados os seguintes critérios de inclusão: publicações gratuitas ou pagas, disponíveis eletronicamente na íntegra, nos idiomas inglês, espanhol, francês e português, com recorte temporal de 10 anos, tratando explicitamente no título e/ou resumo da prevenção das lesões do tipo skin tears no idoso.

Foram excluídos da análise os estudos que apresentavam intervenções em pacientes idosos que possuíam outras afecções de pele, além da lesão skin tear, devido ao fato de dificultarem a avaliação e de poderem interferir nos mecanismos de prevenção e tratamento.

Os resumos dos artigos foram lidos previamente pelos autores em busca de informações que respondessem à questão de pesquisa, sendo excluídas da análise as produções científicas que não continham explicitamente dados sobre os mecanismos de prevenção das lesões do tipo skin tears no idoso e aqueles em duplicidade.

Na PubMed em 12 de Abril de 2014, foi utilizada a estratégia disposta da seguinte forma: "skin tears" and ("older people" or "elderly" or "aged") com um recorte temporal de 10 anos e filtro "Full text available", gerando o código: "skin tears"[All Fields] AND ("older people"[All Fields] OR "elderly"[All Fields] OR "aged"[All Fields]) AND ("loattrfull text"[sb] AND “2003/04/16"[PDat] : "2014/04/12"[PDat]).

Por meio da BVS, em 12 de Abril de 2014, utilizando a estratégia: skin tears and (older people or elderly or aged), foi gerado o seguinte código: skin tears AND (older people OR elderly OR aged) AND year_cluster:(“2012” OR “2009” OR “2013”), com filtro de 2004 a 2013.

Após a seleção, foram iniciadas as traduções e leitura completa dos 12 artigos resultantes, o que possibilitou a coleta sistemática de informações sobre o tema para compor a revisão. Foi utilizado para extração das informações dos artigos selecionados na amostra um instrumento adaptado do protocolo de revisão de Polit, Beck e Hungler ${ }^{18}$, submetido à validação aparente e de conteúdo por especialistas com experiência em pesquisa.

O instrumento utilizado contempla os seguintes dados: título, ano e periódico em que artigo foi publicado, autores, qualificação e origem dos autores, propósito (objetivo) desenho da pesquisa, principais achados relacionados à prevenção e conclusões.

Após a leitura, os artigos selecionados foram organizados em uma pasta e catalogados em ordem numérica crescente de acordo com a cronologia em que eram lidos. Os dados coletados foram analisados, segundo seus conteúdos, por meio da estatística descritiva e quanto à relação das informações com o objeto de interesse em cada estudo.

Posteriormente, os dados extraídos dos artigos foram categorizados em temáticas centrais do delineamento da revisão. A síntese dos dados estratificados dos artigos foi feita contemplando os seguintes aspectos: título, autor, periódico, ano de publicação, objetivos, procedimentos metodológicos e principais resultados. A figura 1 sintetiza o percurso metodológico da revisão integrativa. 
Número de obras sem a associação de descritores
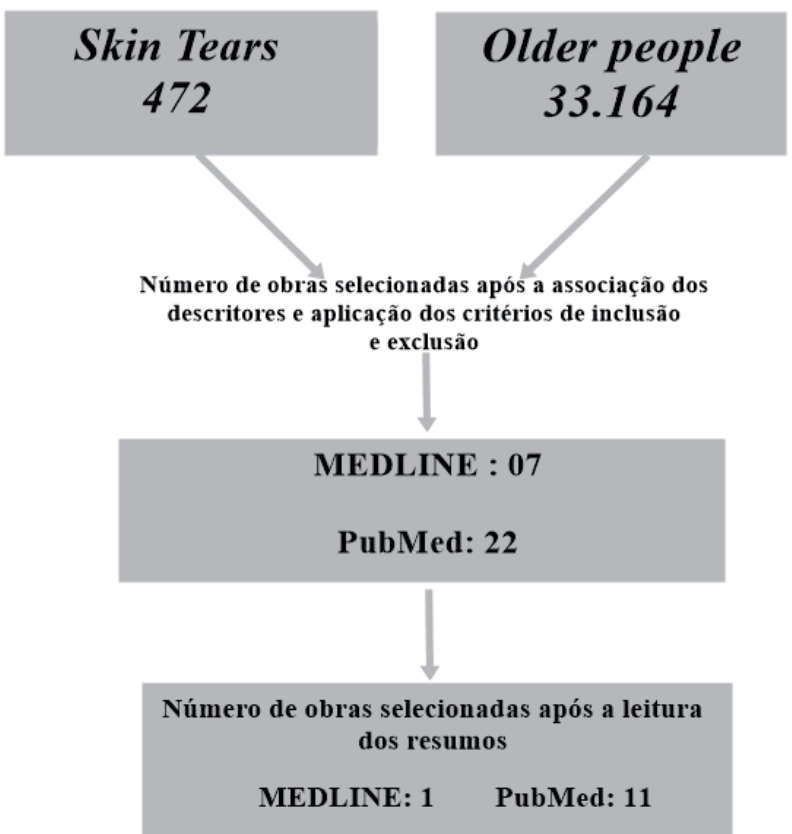

Figura 1 - Fluxograma da busca das obras para compor a revisão integrativa. Rio de Janeiro, 2015.

\section{RESULTADOS E DISCUSSÃO}

$\mathrm{Na}$ base de dados PubMed, foi encontrado o maior número de artigos para compor a revisão. Foram encontrados um total de 22 artigos relacionados, dos quais 11 foram excluídos, sendo 05 por não responderem à questão da pesquisa, e 06 por não possuírem resumos disponíveis para avaliação, alcançando, assim, um total de 11 artigos selecionados para o estudo, como demonstrada nas tabelas 1 e 2 .

Tabela 1 - Artigos selecionados segundo as bases de dados pesquisadas. Rio de Janeiro, 2015.

\begin{tabular}{l|c|c|c|c}
\hline \multirow{2}{*}{$\begin{array}{c}\text { Bases de } \\
\text { dados }\end{array}$} & \multicolumn{2}{|c|}{ Artigos encontrados } & \multicolumn{2}{c}{ Artigos selecionados } \\
\cline { 2 - 5 } & $\mathbf{F a}$ & $\mathbf{F r}(\%)$ & $\mathbf{F a}$ & $\mathrm{Fr}(\%)$ \\
\hline LILACS & 0 & 0 & 0 & 0 \\
\hline IBECS & 0 & 0 & 0 & 0 \\
\hline MEDLINE & 7 & 24,1 & 1 & 8,3 \\
\hline COCHRANE & 0 & 0 & 0 & 0 \\
\hline SciELO & 0 & 0 & 0 & 0 \\
\hline PubMed & 22 & 75,9 & 11 & 91,7 \\
\hline Total & 29 & 100 & 12 & 100 \\
\hline
\end{tabular}

Por meio da BVS, foram acessadas as bases de dados LILACS, IBECS, MEDLINE, COCHRANE e SCIELO, onde foram encontrados 07 artigos relacionados. Destes, 06 foram excluídos, sendo 03 por não responderem à questão da pesquisa, e 03 por duplicação com a seleção da PubMed, alcançando um total de 01 artigo selecionado para o estudo.
Tabela 2 - Artigos selecionados. Rio de Janeiro, 2015.

\begin{tabular}{|c|c|c|}
\hline $\begin{array}{c}\mathrm{N}^{\circ} \\
\text { Artigo }\end{array}$ & Autor (es) & Título do artigo \\
\hline 1 & Stephen-Haynes J. ${ }^{19}$ & $\begin{array}{l}\text { Skin tears: achieving } \\
\text { positive clinical and } \\
\text { financial outcomes. }\end{array}$ \\
\hline 2 & Bianchi J. ${ }^{20}$ & $\begin{array}{l}\text { Preventing, assessing and } \\
\text { managing skin tears. }\end{array}$ \\
\hline 3 & $\begin{array}{l}\text { Lopez V, Dunk AM, } \\
\text { Cubit K, Parke J, } \\
\text { Larkin D, Trudinger } \\
\text { M, et al. }{ }^{21}\end{array}$ & $\begin{array}{l}\text { Skin tear prevention and } \\
\text { management among } \\
\text { patients in the acute aged } \\
\text { care and rehabilitation units } \\
\text { in the Australian Capital } \\
\text { Territory: a best practice } \\
\text { implementation project. }\end{array}$ \\
\hline 4 & $\begin{array}{l}\text { Stephen-Haynes J, } \\
\text { Callaghan R, Bethell } \\
\text { E, Greenwood M. }{ }^{22}\end{array}$ & $\begin{array}{l}\text { The assessment and } \\
\text { management of skin tears in } \\
\text { care homes. }\end{array}$ \\
\hline 5 & $\begin{array}{l}\text { Groom M, Shannon } \\
\text { RJ, Chakravarthy D, } \\
\text { Fleck CA. }{ }^{23}\end{array}$ & $\begin{array}{l}\text { An Evaluation of Costs and } \\
\text { Effects of a Nutrient-Based } \\
\text { Skin Care Program As a } \\
\text { Component of Prevention of } \\
\text { Skin Tears in an Extended } \\
\text { Convalescent Center. }\end{array}$ \\
\hline 6 & $\begin{array}{l}\text { McTigue T, D’Andrea } \\
\text { S, Doyle-Munoz J, } \\
\text { Forrester D.A. } .^{24}\end{array}$ & $\begin{array}{l}\text { Efficacy of a Skin Tear } \\
\text { Education Program. }\end{array}$ \\
\hline 7 & $\begin{array}{l}\text { Xu X, Lau K, Taira } \\
\text { BR, Singer AJ. }{ }^{25}\end{array}$ & $\begin{array}{l}\text { The current management of } \\
\text { skin tears. }\end{array}$ \\
\hline 8 & Battersby L. ${ }^{26}$ & $\begin{array}{l}\text { Exploring best practice in } \\
\text { the management of skin } \\
\text { tears in older people. }\end{array}$ \\
\hline 9 & Roberts MJ. ${ }^{27}$ & $\begin{array}{l}\text { Preventing and Managing } \\
\text { Skin Tears. }\end{array}$ \\
\hline 10 & Brillhart $B .^{28}$ & $\begin{array}{l}\text { Pressure Sore and Skin Tears } \\
\text { Prevention and Treatment } \\
\text { During a 10-Month Program. }\end{array}$ \\
\hline 11 & $\begin{array}{l}\text { Hodgkinson B, Nay } \\
R^{29}\end{array}$ & $\begin{array}{l}\text { Effectiveness of topical skin } \\
\text { care provided in aged care } \\
\text { facilities. }\end{array}$ \\
\hline 12 & $\begin{array}{l}\text { Holmes RF, } \\
\text { Davidson MW, } \\
\text { Thompson BJ, } \\
\text { Kelechi TJ.30 }\end{array}$ & $\begin{array}{l}\text { Skin Tears: Care and } \\
\text { Management of the Older } \\
\text { Adult at Home. }\end{array}$ \\
\hline
\end{tabular}

Das publicações selecionadas na amostra da revisão, todas foram desenvolvidas em outros países, sendo 06 (50\%) nos Estados Unidos, 04 (33,3\%) na Inglaterra e 02 (16,7\%) na Austrália. Nenhum resultado foi encontrado no Brasil, de acordo com os critérios de seleção e exclusão da amostra, o que ratifica o quanto a temática é pouco discutida no âmbito nacional. Não obstante ao ineditismo da temática ou à dificuldade de se encontrar publicações nacionais, a lesão é muito frequente, porém, até o momento, ela tem sido genericamente denominada laceração, a 
exemplo da literatura especializada estrangeira ${ }^{10}$. A falta de uniformização dos termos e definições relacionadas às lesões do tipo skin tears é um fator que contribui para dificultar a busca e disseminação das publicações disponíveis nas bases de dados.

Em relação ao perfil profissional dos autores dos estudos selecionados, a maior parte deles eram enfermeiros (73,5\%), 04 eram médicos (11,8\%), e os demais $(14,7 \%)$ eram biólogos ou outros profissionais da área da saúde.

São notórios o envolvimento e a responsabilidade profissional, ética e legal do enfermeiro na prestação de cuidados para manutenção da integridade da pele. Com isto, fica evidente a necessidade da pesquisa e da geração de conhecimentos acerca da estrutura e função da pele, como fundamento tanto da prevenção como dos cuidados eficazes para manutenção da pele íntegra ${ }^{7}$.

As pesquisas sobre tratamento de feridas recebem grande destaque nas publicações de enfermagem. 0 mesmo, contudo, não ocorre nas publicações médicas, demonstrando que a responsabilidade do tratamento e prevenção de feridas vêm sendo atribuída ao enfermeiro, devendo ele avaliar a lesão e prescrever o tratamento mais adequado, além de orientar e supervisionar a equipe de enfermagem na execução do curativo. É necessário que o enfermeiro perceba que essas competências são intrínsecas ao seu cotidiano ${ }^{31}$.

Foi encontrada nos resultados destes estudos uma série de medidas preventivas que, em conjunto, podem ser eficazes para evitar o surgimento da lesão do tipo skin tear na pele senil. 0 enfermeiro deve estar em constante processo de atualização, para apropriar-se de conhecimentos relacionados à assistência de enfermagem, adequar-se às suas finalidades essenciais e se motivar na busca da melhoria da qualidade. A prevenção necessita de uma estratégia individual precisa, o que exige muita dedicação e preparo do profissional, para uma intervenção de enfermagem ajustada e eficaz ${ }^{32}$.

Os conhecimentos que fornecem subsídios para o cuidar do idoso e de seu cuidador familiar incluem o entendimento das necessidades humanas básicas, bem como adaptações e mudanças que ocorrem ao longo da vida que, por sua vez, apresentam dimensões biológica, psicológica, social, cultural e espiritual. Ao cuidar do ser idoso e de seu cuidador, não basta focar ações na patologia, mas priorizar a promoção, manutenção e recuperação da saúde. Respeitar a independência e propiciar a participação do sujeito idoso e de seu cuidador familiar no processo de cuidado, portanto, pode favorecer a assistência qualificada ${ }^{33}$.

Uma das alternativas mais importantes para assegurar a autonomia e independência do idoso, como também o envelhecer saudável, é a ação educativa para esta parcela da sociedade. Porém, infelizmente, o modelo assistencial privilegia as ações curativas, centradas na atenção médica, desconsiderando o papel ativo que o sujeito cuidado pode desempenhar ${ }^{33}$.
Ao identificar as atividades desenvolvidas pelos profissionais de enfermagem no desempenho de suas funções, verifica-se a necessidade de reafirmar a questão educativa como compromisso com o crescimento pessoal e profissional, visando melhorar a qualidade da prática profissional ${ }^{34}$. Observa-se entre os resultados do estudo que as medidas de prevenção das lesões do tipo skin tears estão, em boa parte, associadas às ações educativas, tanto no âmbito dos profissionais que cuidam da lesão como entre os pacientes, cuidadores e familiares.

Conhecer e entender os mecanismos causais da lesão e as intervenções no ambiente familiar, incentivar o idoso, os cuidadores e a família a participarem do desenvolvimento de medidas preventivas, ratificando a autonomia do sujeito neste contexto do envelhecimento, é imperativo para reduzir a incidência da lesão, conforme foi apresentado nos resultados e no quadro 1.

Quadro 1 - Categorização e síntese das principais recomendações da revisão. Rio de Janeiro, 2015.

\begin{tabular}{|c|c|}
\hline Categoria & Achados \\
\hline $\begin{array}{l}\text { Mantendo a } \\
\text { homeostase } \\
\text { cutânea no } \\
\text { processo do } \\
\text { envelhecimento }\end{array}$ & $\begin{array}{l}\text { - Manter bom estado nutricional; } \\
\text { - Manter equilíbrio hídrico; } \\
\text { - Usar diariamente, ao menos 2x ao dia, } \\
\text { hidratantes de pele; } \\
\text { - Evitar banhos frequentes e usar sabão } \\
\text { emoliente de ph neutro; }\end{array}$ \\
\hline $\begin{array}{l}\text { Evitando } \\
\text { acidentes com a } \\
\text { pele no contexto } \\
\text { da senilidade }\end{array}$ & $\begin{array}{l}\text { - Usar calçados antiderrapantes; } \\
\text { - Retirar excesso de móveis e tapetes; } \\
\text { - Iluminação adequada; } \\
\text { - Manter unhas de pacientes e } \\
\text { cuidadores aparadas; } \\
\text { - Evitar usar adesivos e ter cautela na } \\
\text { remoção; } \\
\text { - Manipular e limpar suavemente a } \\
\text { pele do idoso; }\end{array}$ \\
\hline $\begin{array}{l}\text { Protegendo a pele } \\
\text { senil do trauma } \\
\text { mecânico }\end{array}$ & $\begin{array}{l}\text { - Usar calças, camisas de manga longa } \\
\text { e meias; } \\
\text { - Usar técnicas adequadas de } \\
\text { posicionamento, transferência e } \\
\text { movimentação no leito; } \\
\text { - Acolchoar utensílios que possam } \\
\text { provocar pressão, atrito e cisalhamento; }\end{array}$ \\
\hline $\begin{array}{l}\text { Sistematizando } \\
\text { e educando para } \\
\text { o cuidado com a } \\
\text { pele do idoso }\end{array}$ & $\begin{array}{l}\text { - Educação permanente dos } \\
\text { profissionais de e cuidadores; } \\
\text { - Envolver o paciente e a família na } \\
\text { prevenção; } \\
\text { - Avaliar periodicamente o risco } \\
\text { para a lesão e manutenção da } \\
\text { sustentabilidade da pele; } \\
\text { - Usar protocolos sistemáticos de } \\
\text { prevenção; }\end{array}$ \\
\hline
\end{tabular}




\section{CONCLUSÕES}

A lesão do tipo skin tear, para além do seu ineditismo enquanto tema de estudo em âmbito nacional, ainda é envolvida pela falta de uniformização dos termos relacionados, dificultando a busca das publicações nas bases de dados, uma vez que a criação de uma estratégia satisfatória implica a necessidade de um consenso de palavras-chave e descritores relacionados ao tema. Considerando esta limitação inerente ao tema até o momento, esta revisão integrativa possibilitou a coleta sistemática de informações sobre a prevenção da lesão no idoso a partir de estudos estadunidenses, ingleses e australianos, dentre os quais o maior número de autorias pertence à categoria de enfermeiros.

Para a prevenção da lesão do tipo skin tear no idoso, fazse necessária atenção a quatro esferas do cuidado: manter a homeostase cutânea no processo do envelhecimento atentando para nutrição e hidratação adequada; evitar acidentes com a pele no contexto da senilidade gerindo um ambiente seguro e escolhendo dispositivos adequados para o contato com a pele; proteger a pele senil do trauma mecânico com o uso de roupas e protetores adequados; sistematizar e educar para o cuidado com a pele do idoso nos âmbitos da educação permanente e em saúde.

Em seus achados, as recomendações oferecidas pela revisão da pesquisa dão suporte às decisões na confecção do plano de cuidados direcionado à prevenção da lesão no idoso, de modo que essas recomendações sejam incorporadas à prática de enfermagem nos diferentes níveis da atenção. As quatro esferas do cuidado sintetizadas por meio da análise dos resultados obtidos por este estudo são de extrema importância e pertinência quando pensamos no envelhecimento da população brasileira e a necessidade de uma assistência de enfermagem adequada e eficaz no que tange a prevenção das lesões mais prevalentes neste grupo.

A falta de estudos brasileiros e a prevalência de estudos metodologicamente inconsistentes, no que corresponde à oferta de evidências sobre a temática, deixam algumas lacunas que foram identificadas por este estudo, sinalizando a necessidade do desenvolvimento de novos estudos no âmbito nacional e internacional, especialmente clínicos, para fundamentar a prática de prevenção das skin tears por meio de evidências científicas mais robustas.

\section{REFERÊNCIAS}

1. Payne R, Martin M. The epidemiology and management of skin tears in older adults. Ostomy Wound Manage. 1990;26:26-37.

2. Bryant R, Rolstand B. Examining threats to skin integrity. Ostomy Wound Manage. 2001;47(6):18-27.

3. McGough-Csarny J, Kopac CA. Skin tears in institutionalized elderly: an epidemical study. Ostomy Wound Manage. 1998:44(3 suppl):S14-25.

4. LeBlanc K, Baranoski S. Skin tears: state of the science: consensus statements for the prevention, prediction, assessment, and treatment of skin tears. Adv Skin Wound Care [internet]. 2011[acesso em 2013 Mai 09];24(9):2-15. Disponível em: http://www.skintears.org/Consensus-Statements/. Acessado em: 19 Out 2015

5. Bank D, Nix D. Preventing skin tears in a nursing and rehabilitation centers: an interdisciplinary effort. Ostomy Wound Manage. 2006;52(9):38-46.

6. LeBlanc K, Baranoski S. Prevention and management of skin tears. Adv Skin Wound Care. 2009;22(7):325-332.

7. Fernandes AAL, Darlen SDV, Linhares CAM, Dire GF. O enfermeiro atuando na prevenção das úlceras de pressão. Enfermería Global [internet]. 2008 Jun [acesso em 2013 Mai 15];(13):1-15.

8. Arnold Jr HL, Odom RB, James WD. A pele: estrutura básica e função. Doenças básicas da pele de Andrews: Dermatologia clínica. 8ª . São Paulo (SP): Manole; 1994. p.1-14.

9. Fenske NA, Lober CW. Skin changes of aging: pathological implications. Geriatrics. 1990;45(3):27-35.

10. Strazzieri-Pulido KC. Adaptação cultural e validação do instrumento "star skin tear classification system", para a língua portuguesa no Brasil [dissertação]. São Paulo (SP): Escola de Enfermagem da Universidade de São Paulo; 2010.

11. Souza DMST, Santos VLCG. Úlceras por pressão e envelhecimento. Rev Estima [internet]. 2006 [acesso em 2013 Mai 15];4(1):36-44.

12. Krasner D. Skin tears: understanding problem leads to prevention, proper care [latter]. Feature Wound Care. 2010 Apr;(30).

13. Payne R, Martin M. Defining and classifying skin tears: need for a common language. Ostomy Wound Manage. 1993;39(5):1620.

14. Instituto Brasileiro de Geografia e Estatística - IBGE [acesso em 2013 mai 05]. Disponível em: http://saladeimprensa.ibge.gov. $\mathrm{br} /$ noticias?view=noticia \&id=1 \&busca=1 \&idnoticia=1272.

15. Mendes KDS, Silveira RCCP, Galvão CM. Revisão Integrativa: método de pesquisa para a incorporação de evidências na saúde e na enfermagem. Texto \& Contexto Enferm. 2008 OutDez;17(4):758-64.

16. Roman AR, Friedlander MR. Revisão integrativa de pesquisa aplicada à enfermagem. Cogitare Enferm. 1998 JulDez;3(2):109-12.

17. Souza MT, Silva MD, Carvalho R. Revisão integrativa: o que é e como fazer. Einstein [internet]. 2010[acesso em 2013 Mai 19];8(1 Pt 1):102-6.

18. Polit DF, Beck CT, Hungler BP. Study guide to accompany essentials of nursing research: methods, appraisals, and utilization. 5th.ed. Philadelphia: Lippincott; 2001c.

19. Stephen-Haynes J. Skin tears: achieving positive clinical and financial outcomes. Wound Care. 2012 Mar:Suppl:S6-16.

20. Bianchi J. Preventing, assessing and managing skin tears. Nurs Times. 2012;108(13):12-16.

21. Lopez V, Dunk AM, Cubit K, Parke J, Larkin D, Trudinger M, et al; Skin tear prevention and management among patients in the acute aged care and rehabilitation units in the Australian Capital Territory: a best practice implementation project. Joanna Briggs Institute. Int J Evid Based Healthc. 2011;9(4):429-34. 
22. Stephen-Haynes J, Callaghan R, Bethell E, Greenwood M. The assessment and management of skin tears in care homes. $\mathrm{Br} J$ Nurs. 2011;20(11 suppl):S12-16.

23. Groom M, Shannon RJ, Chakravarthy D, Fleck CA . An evaluation of costs and effects of a nutrient-based skin care program as a component of prevention of skin tears in an extended convalescent center. J Wound Ostomy Continence Nurs. 2010 Jan-Feb;37(1):46-51. Erratum in: J Wound Ostomy Continence Nurs. 2010 Mar-Apr;37(2):128.

24. McTigue T, D’Andrea S, Doyle-Munoz J, Forrester D.A. Efficacy of a skin tear education program.J Wound Ostomy Continence Nurs. 2009 Sep-Oct;36(5):486-92.

25. Xu X, Lau K, Taira BR, Singer AJ. The current management of skin tears. Am J Emerg Med. 2009 Jul;27(6):729-33.

26. Battersby L. Exploring best practice in the management of skin tears in older people. Nurs Times. 2009 Apr 28-May 4;105(16):22-6.

27. Roberts MJ. Preventing and managing skin tears. J Wound Ostomy Continence Nurs. 2007 May-Jun; 34(3):256-9.

28. Brillhart B. Pressure sore and skin tears prevention and treatment during a 10-month program. Rehabil Nurs. 2005 May-Jun;30(3):85-91.

29. Hodgkinson B, Nay R. Effectiveness of topical skin care provided in aged care facilities. Int J Evid Based Healthc. 2005 May;3(4):65-101.

30. Holmes RF, Davidson MW, Thompson BJ, Kelechi TJ. Skin tears: care and management of the older adult at home. Home Healthc Nurse. 2013 Feb;31(2):101-3.

31. Ferreira AM, Bogamil DDD, Tormena PC. O enfermeiro e o tratamento de feridas: em busca da autonomia do cuidado. Arq Ciênc Saúde [internet]. 2008 Jul-Set [acesso em 2013 Mai 25];15(3):105-9.

32. Rodrigues MM, Souza e Souza M, Silva JL. Sistematização da assistência de enfermagem na prevenção da lesão tecidual por pressão. Cogitare Enferm. 2008 Out-Dez;13(4):566-75.

33. Martins JJ, Albuquerque GL, Nascimento ERP, Barra DCC, Souza WGA, Pacheco WNS, et al. Necessidade de educação em saúde dos cuidadores de pessoas idosas no domicílio. Texto \& Contexto Enferm. 2007 Abr-Jun;16(2):254-62.

34. Paschoal AS, Mantovani MF, Lacerda MR. A educação permanente em enfermagem: subsídios para a prática profissional. Rev Gaúcha Enferm [internet]. 2006 Set [acesso em 2013 Mai 25];27(3):336-43. 